SU-GP-95-5-1

WISC-MILW-95-TH-16

PP96-40

gr-qc/9511023

\title{
Complex actions in two-dimensional topology change
}

\author{
Jorma Louko* \\ Department of Physics, University of Wisconsin-Milwaukee, \\ P.O. Box 413, Milwaukee, Wisconsin 53201, USA \\ and \\ Department of Physics, University of Maryland, \\ College Park, Maryland 20742-4111, USA ${ }^{\dagger}$ \\ Rafael D. Sorkin $\ddagger$ \\ Department of Physics, Syracuse University, Syracuse, New York 13244-1130, USA \\ and \\ Instituto de Ciencias Nucleares, UNAM, A. Postal 70-543, D.F. 04510, Mexico \\ (Revised version, September 1996. Published in Class. Quantum Grav. 14 (1997) 179-203)
}

\begin{abstract}
We investigate topology change in $(1+1)$ dimensions by analyzing the scalarcurvature action $\frac{1}{2} \int R d V$ at the points of metric-degeneration that (with minor exceptions) any nontrivial Lorentzian cobordism necessarily possesses. In two dimensions any cobordism can be built up as a combination of only two elementary types, the "yarmulke" and the "trousers." For each of these elementary cobordisms, we consider a family of Morse-theory inspired Lorentzian metrics that vanish smoothly at a single point, resulting in a conical-type singularity there. In the yarmulke case, the distinguished point is analogous to a cosmological initial (or final) singularity, with the spacetime as a whole being obtained from one causal region of Misner space by adjoining a single point. In the trousers case, the distinguished point is a "crotch singularity" that signals a change in the spacetime topology (this being also the fundamental
\end{abstract}

\footnotetext{
${ }^{*}$ On leave of absence from Department of Physics, University of Helsinki. Electronic address: louko@wam.umd.edu

${ }^{\dagger}$ Present address.

${ }^{\ddagger}$ Electronic address: rdsorkin@mailbox.syr.edu
} 
vertex of string theory, if one makes that interpretation). We regularize the metrics by adding a small imaginary part whose sign is fixed to be positive by the condition that it lead to a convergent scalar field path integral on the regularized spacetime. As the regulator is removed, the scalar density $\frac{1}{2} \sqrt{-g} R$ approaches a delta-function whose strength is complex: for the yarmulke family the strength is $\beta-2 \pi i$, where $\beta$ is the rapidity parameter of the associated Misner space; for the trousers family it is simply $+2 \pi i$. This implies that in the path integral over spacetime metrics for Einstein gravity in three or more spacetime dimensions, topology change via a crotch singularity is exponentially suppressed, whereas appearance or disappearance of a universe via a yarmulke singularity is exponentially enhanced. We also contrast these results with the situation in a vielbein-cum-connection formulation of Einstein gravity.

Pacs: $04.20 . \mathrm{Fy}, 04.60 . \mathrm{Kz}, 04.60 . \mathrm{Gw}$ 


\section{INTRODUCTION}

General relativity in $D \geq 3$ spacetime dimensions is most commonly formulated as a generally covariant, nonlinear field theory for the spacetime metric [1]3]. One starts by assuming the (Lorentzian) metric $g_{a b}$ to be smooth (say, $C^{2}$ or $C^{\infty}$ ) and invertible. The Riemann tensor $R_{b c d}^{a}$ and the Einstein tensor $G_{a b}=R_{a b}-\frac{1}{2} R g_{a b}$ are then well defined, and the gravitational field equations (with matter) read

$$
G_{a b}=\kappa T_{a b}
$$

where $T_{a b}$ is the stress-energy tensor of the matter and $\kappa$ is the gravitational constant. Equivalently, the classical solutions can be defined as the critical points of an action functional whose gravitational part reads

$$
S=\frac{1}{2 \kappa} \int d^{D} x \sqrt{-g} R+\text { (boundary terms) }
$$

where the variation is taken within smooth invertible metrics (and smooth matter fields) with appropriate boundary conditions. A variational formulation is particularly natural if one wishes to view the theory as the classical limit of a quantum theory of gravity, where it is not the field equations, but rather the action that plays the central role.

However, the assumption of a smooth, invertible metric is too strong to accommodate certain situations of physical interest. One such situation, by now well understood, arises with an idealized matter source whose $T_{a b}$ is a distribution concentrated on a hypersurface of codimension one. The metric is then invertible and $C^{0}$ everywhere, and smooth outside the timelike $(D-1)$-dimensional world hypersurface of the matter, but it fails to be $C^{1}$ across this hypersurface. For $D=4$, this situation reduces to the familiar case of an infinitely thin matter shell. The Einstein equations (1.1) for such spacetimes can be given a distributional interpretation [4] that is equivalent to Israel's junction condition formalism [5], and also the Einstein-Hilbert action (1.2) can be readily generalized to such spacetimes in a manner that reproduces the junction condition formalism from a variational principle [6]. These spacetimes belong to the wider class of invertible but nonsmooth metrics for which Geroch and Traschen showed that the Einstein equations (1.1) have a well-defined distributional interpretation [4]. There has also been interest in metrics that incorporate a signature change on a surface of codimension 1; see for example Refs. [0] 15] and the references therein.

The main purpose of the present paper is to extend the definition of the Einstein-Hilbert action (1.2) to incorporate certain metrics that are smooth but not everywhere invertible.

\footnotetext{
${ }^{1}$ We use units in which $\hbar=c=1$, but we keep the gravitational constant $\kappa$. In four dimensions $\kappa=8 \pi G$, where $G$ is Newton's constant.
} 
The prime motivation for considering such metrics comes from topology change. The requirement of a nondegenerate Lorentzian metric renders topology change in two spacetime dimensions essentially impossible, while in higher dimensions this requirement leads to causality violations [16.[17, or to apparently unwarranted restrictions on the allowed transitions, such as the exclusion of Kaluza-Klein monopole pair production [18.19]. However, there exists a class of metrics for which the regularity assumptions are relaxed in a relatively mild manner, but one still broad enough that metrics of the resulting type can exist on any (smooth) cobordism in any dimension. Moreover, these metrics respect causality (they are compatible with a global causal ordering of the points of the manifold), and they are free of any other apparent pathology.

The inclusion of these metrics, in which the assumption of global invertibility has been relaxed, renders topology change kinematically possible, and the issue of topology change becomes a question about the dynamics of the theory. Within path-integral quantization, one must ask how such metrics contribute to the gravitational path integrals that give transition amplitudes for topology change. At the classical level, one can ask whether such metrics are (in an appropriate sense) critical points of the generalized Einstein-Hilbert action, this being the criterion for them to make a non-negligible contribution to the quantum amplitude in the classical limit.

The metrics discussed in [16,17] fail to be invertible only at isolated degenerate points. In this paper we will consider the two-dimensional special case of these metrics, as well as higher-dimensional metrics that locally look like products of the two-dimensional metrics with a flat metric. Thus, a characteristic feature of our metrics will be that the degeneracy will be concentrated on a submanifold of codimension two. Although the metric itself will be smooth everywhere, the geometry it describes can be understood as having a conical-type singularity at the submanifold of degeneracy, and we shall refer to the locus of degeneracy in this sense as a singularity. Singularities of exactly this type appear also in Regge calculus, as the support of the spacetime curvature [20]. We note that degeneration of the metric at submanifolds of positive codimension can be viewed as a particularly mild form of singularity, if one reflects that the path-integral measure is expected to be supported entirely on nonsmooth configurations.

When the conically singular submanifold is timelike, or when the whole spacetime is taken to have Euclidean signature, it is known that the spacetime can be understood as having a distributional curvature at the singularity, in a sense that we shall discuss in more detail below (see also Refs. [20 26]): the Ricci scalar density has at the singularity a delta-function whose strength is twice the defect angle. This is the case with the well-known spacetimes of idealized cosmic strings for $D=4$ [27 29], and spacetimes with massive point particles for $D=3$ [30,31]. The Einstein-Hilbert action (1.2) can be readily extended to metrics of this type [6,20,32 35], with the result that the singular spacetimes are not critical points of the action. In the vacuum theory defined in terms of the Einstein-Hilbert variational principle thus generalized, one therefore concludes that these singular spacetimes are not classical 
solutions, but one also finds that the degrees of freedom associated with the singularity may have significance when one quantizes the theory by path-integral methods [6, 20, 32, 35]. Note, however, that extending the variational principle or the field equations to include an idealized matter source that would make these singular spacetimes classical solutions has proved difficult [4,36]. For example, for an idealized cosmic string in $D=4$, with $T_{a b}$ a delta-function concentrated on the world sheet of the string, there is no unambiguous relation between the defect angle and the linear mass density of the string 36.

Our main aim is to perform a similar analysis in the case where the spacetime is Lorentzian and the conically singular submanifold is spacelike. As in the Riemannian case, we will find that conically singular metrics are not extrema of the action. But we also find a surprising new feature: the action itself is no longer real but complex.

To begin, we consider two one-parameter families of Morse theory inspired metrics on $\mathbb{R}^{2}$. The metrics are everywhere $C^{\infty}$ (and even analytic), and they turn out to be flat with Lorentz signature everywhere except at a single point, at which they vanish. The metrics of the first family describe "yarmulke" spacetimes that can be obtained from one causal domain of Misner space [2] by adjoining a single point, the singularity at the added point being analogous to a cosmological initial (or final) singularity. The metrics of the second family describe a Lorentzian "trousers" spacetime near the "crotch singularity" 37 41.

We wish to give a meaning to the action, and secondarily the Ricci scalar density, on these two-dimensional spacetimes. To do so, we regularize the metrics by adding a small positive imaginary part, the sign of the regulator following from the requirement that a scalar field path integral on the regularized spacetime should be (formally) convergent. Upon taking the regulator to zero, we find that the scalar density $\frac{1}{2} \sqrt{-g} R$ converges to a delta-function at the singularity. The strength of the delta-function turns out to be complex: $\beta-2 \pi i$ for the yarmulke singularity, where $\beta$ is the rapidity parameter of the Misner space, and $+2 \pi i$ for the crotch singularity. This agrees with results obtained from a Regge calculus description of these singular spacetimes [16] (see also the appendix of Ref. [20]).

Turning to $D \geq 3$ dimensions, we then consider spacetimes that are products of our conical two-dimensional spacetimes with a $(D-2)$-dimensional compact flat space. If we take the Einstein-Hilbert action for such spacetimes to be defined via the same limiting process, it is immediately seen that the action is not stationary under variations in the $(D-2)$-volume, and we shall argue that this non-stationarity remains even after the global product form of the metric is relaxed and the variations become properly localized. In the theory that is defined in terms of the Einstein-Hilbert action thus extended, our singular spacetimes are therefore not classical solutions: topology change via the yarmulke and trousers cobordisms is classically forbidden.

Within a path-integral quantized theory, the question of topology change via our metrics amounts to asking how these metrics contribute to the gravitational path integral. The above results imply that the contribution from the trousers spacetimes is suppressed by an exponential factor, over and above what one expects from the fact that these spacetimes are 
not stationary points of the action. Topology change via the trousers cobordism is therefore exponentially suppressed; in $3+1$ dimensions the suppression factor is $\exp (-A / 4 G)$, where $A$ is the area of the two flat dimensions. In contrast, the contribution from the yarmulke spacetimes is enhanced by the same exponential factor. One might take this as evidence for an exponential enhancement of the creation (or annihilation) of a component of spacetime via the yarmulke cobordism. However, one would need to balance such an exponential enhancement against the suppression coming from the fact that the yarmulke spacetimes are not stationary points of the action.

These conclusions in $D \geq 3$ dimensions also provide an interesting contrast to the vielbein-cum-connection formulation of Einstein gravity, within which the trousers spacetime solves the field equations, and even appears to represent a stationary point of the action in the relevant sense. If this is indeed so, then it would seem that the metric and vielbein-connection formulations must be regarded as physically inequivalent.

The paper is organized as follows. In Section \ we introduce the two families of regularized metrics on $\mathbb{R}^{2}$, discuss their properties, and compute the distributional limit of the Ricci scalar density as the regulator is taken to zero. For the sake of comparison, we carry the analysis through in both the Lorentzian and Euclidean cases; in the Euclidean case the regulator can be taken real, and we reproduce the familiar result for a Euclidean conical singularity. In Sections $[1]-\nabla$ we shall present evidence to the effect that our results are independent of the details of the regularization, and that they should even be insensitive to the choice of the differentiable structure at the singularity: one set of arguments relies on a suitably generalized Gauss-Bonnet theorem, another on Regge calculus. Section V1 contains a summary and discussion. Appendix A compares our method to the vielbeincum-connection formulation for the $(2+1)$-dimensional spacetime that is the product of the time-axis with the "double cover" conical metric. Appendix B presents a similar comparison for the "hourglass" spacetime of Refs. 42,43.

\section{REGULARIZED METRICS AND THE RICCI SCALAR DENSITY}

As mentioned in the Introduction, a primary reason for our interest in conical-type singularities is their role in two-dimensional topology change. In this context they fit into a general scheme for endowing any (smooth) topological cobordism? with a Lorentzian metric. Although a globally regular Lorentzian metric will exist in some cobordisms, it will not exist in general, and when it does exist, it necessarily contains time loops (assuming a time-oriented metric on a compact, non-product cobordism with spacelike boundaries). Even accepting these time loops, one still cannot find Lorentzian metrics for certain cases

\footnotetext{
${ }^{2}$ By a topological cobordism, we just mean a compact (say) manifold with boundary, regarded as mediating a transition between different spacetime topologies.
} 
where, on physical grounds, one would want them to exist, such as on the natural cobordism for mediating the pair creation of Kaluza-Klein monopoles in five dimensions. For a general discussion, see for example Refs. [18,19,44] and the references therein. In contrast, there always exists a Morse function [45] $f$ on any cobordism, as well as a positive-definite, Riemannian metric $h_{a b}$. From such a pair one can construct a metric that is almost Lorentzian (in the sense of being smooth with Lorentzian signature everywhere except at the critical points of $f$ ), and further has a well-defined causal structure for which $f$ furnishes a "time function" 16, 17. The resulting geometry can thus be taken to contribute to the path integral amplitude for the topology change which the cobordism mediates. Specifically, the metric in question has the form

$$
g_{a b}=\left(\partial_{c} f\right)\left(\partial_{d} f\right) h^{c d} h_{a b}-\zeta\left(\partial_{a} f\right)\left(\partial_{b} f\right)
$$

where the parameter $\zeta$ must satisfy $\zeta>1$ in order that the signature be Lorentzian. The metrics we will study in the present paper belong to this family with $h_{a b}$ chosen as flat and with $f$ a quadratic polynomial in Cartesian coordinates for $h_{a b}$. By a remarkable accident, (2.1) then turns out (in two dimensions) to be flat everywhere except at the origin 46.

Let $(x, y)$ be a global coordinate chart on $\mathbb{R}^{2}$. We shall consider on $\mathbb{R}^{2}$ the metric [16,17]

$$
g_{a b}=\left(\partial_{c} f\right)\left(\partial_{d} f\right) \delta^{c d} \delta_{a b}-\zeta\left(\partial_{a} f\right)\left(\partial_{b} f\right)+\gamma \delta_{a b}
$$

where $f=\frac{1}{2}\left(x^{2}+\epsilon y^{2}\right)$ is the Morse function, $\epsilon= \pm 1$ is essentially the Morse index of $f$, and $\zeta$ and $\gamma$ are parameters. We take $\gamma \in \mathbb{C}$, and $\zeta \in \mathbb{R} \backslash\{1\}$. In the chart $(x, y)$ we have then

$$
d s^{2}=g_{a b} d x^{a} d x^{b}=\left(x^{2}+y^{2}+\gamma\right)\left(d x^{2}+d y^{2}\right)-\zeta(x d x+\epsilon y d y)^{2}
$$

This metric is $C^{\infty}$ (and even analytic) in the differentiable structure determined by the chart $(x, y)$, but it need not be everywhere invertible. Our aim is to investigate the limit $\gamma=0$, in which the metric is invertible everywhere except at the point $x=y=0$. We shall treat $\gamma$ as a regulator, chosen to make the metric invertible, and we will examine the curvature in the limit $\gamma \rightarrow 0$. There are four qualitatively different cases depending on $\epsilon$ and the sign of $\zeta-1$. We shall devote a separate subsection to each case.

\section{A. General Euclidean conical singularity}

We take first $\epsilon=1, \zeta<1$. When $\gamma=0$, the metric (2.3) has signature $(++)$ everywhere except at $x=y=0$. To make the geometry more transparent, we perform for $x^{2}+y^{2}>0$ the coordinate transformation

$$
\begin{aligned}
& x=\sqrt{r} \cos \varphi, \\
& y=\sqrt{r} \sin \varphi,
\end{aligned}
$$


where $r>0$. We understand $\varphi$ to be periodically identified with period $2 \pi,(r, \varphi) \sim$ $(r, \varphi+2 \pi)$. The metric reads then

$$
d s^{2}=\frac{1}{4}(1-\zeta) d r^{2}+r^{2} d \varphi^{2}
$$

which is recognizable as the metric of a cone with defect angle $2 \pi\left(1-2(1-\zeta)^{-1 / 2}\right)$. Note that when $\zeta=-3$ the defect angle vanishes and the cone reduces to a plane.

We now take the regulator $\gamma$ to be positive. The metric (2.3) has then signature $(++)$ everywhere, including the origin. With the notation

$$
\begin{aligned}
N & =\sqrt{(1-\zeta) \rho^{2}+\gamma}, \\
a & =\rho \sqrt{\rho^{2}+\gamma},
\end{aligned}
$$

where $\rho=\sqrt{x^{2}+y^{2}}=\sqrt{r}$, the Ricci scalar takes on the appearance

$$
R=-\frac{2}{a N}\left(\frac{a^{\prime}}{N}\right)^{\prime}
$$

where the prime denotes derivative with respect to $\rho$. The volume element of the metric is given by

$$
\sqrt{g} d^{2} x=\rho^{-1} a N d x d y .
$$

We wish to examine the scalar density $\frac{1}{2} \sqrt{g} R$ in the limit $\gamma \rightarrow 0$. For this purpose, let $\Phi(x, y)$ be a test function on $\mathbb{R}^{2}$ [47, [ ] and consider the integral of the density $\frac{1}{2} \sqrt{g} R \Phi$. Using the polar coordinates

$$
\begin{aligned}
& x=\rho \cos \varphi, \\
& y=\rho \sin \varphi,
\end{aligned}
$$

and writing

$$
\bar{\Phi}(\rho)=(2 \pi)^{-1} \int_{0}^{2 \pi} d \varphi \Phi(\rho \cos \varphi, \rho \sin \varphi)
$$

we obtain

$$
\begin{aligned}
\frac{1}{2} \int_{\mathbb{R}^{2}} d^{2} x \sqrt{g} R \Phi & =-\int_{\mathbb{R}^{2}} d x d y \frac{1}{\rho}\left(\frac{a^{\prime}}{N}\right)^{\prime} \Phi \\
& =-2 \pi \int_{0}^{\infty} d \rho\left(\frac{a^{\prime}}{N}\right)^{\prime} \bar{\Phi}(\rho) \\
& =-2 \pi \int_{0}^{\infty} d \rho\left(\frac{a^{\prime}}{N}-\frac{2}{\sqrt{1-\zeta}}\right)^{\prime} \bar{\Phi}(\rho) \\
& =2 \pi\left(1-\frac{2}{\sqrt{1-\zeta}}\right) \bar{\Phi}(0)+2 \pi \int_{0}^{\infty} d \rho\left(\frac{a^{\prime}}{N}-\frac{2}{\sqrt{1-\zeta}}\right) \bar{\Phi}^{\prime}(\rho)
\end{aligned}
$$

\footnotetext{
${ }^{3}$ The distinction between test functions of rapid decrease and test functions of compact support will be irrelevant here.
} 
where in the last step we have integrated by parts and used the fact that $a^{\prime} / N \rightarrow 1$ as $\rho \rightarrow 0$. Note that $\bar{\Phi}^{\prime}(\rho)$ vanishes at $\rho=0$. Now, in the limit $\gamma \rightarrow 0$, the integral term on the last line of $(2.11)$ vanishes by dominated convergence. As $\bar{\Phi}(0)=\Phi(0,0)$, this means that the $\gamma \rightarrow 0$ limitf of the density $\frac{1}{2} \sqrt{g} R$ is the distribution

$$
\frac{1}{2} \sqrt{g} R=2 \pi\left(1-\frac{2}{\sqrt{1-\zeta}}\right) \delta_{2}(x, y),
$$

where $\delta_{2}(x, y)$ stands for the two-dimensional delta-function concentrated at $x=y=0$. The coefficient of the delta-function in (2.12) is precisely the defect angle. This is the well-known result for a conical singularity [20].

Note that in (2.12) the left hand side is understood as a single entity: we are not attempting here to give a distributional meaning to the Ricci scalar $R$ as such. We shall return to this issue in Sections $[\mathrm{II}$ and IV.

\section{B. Lorentzian yarmulke singularity}

We take next $\epsilon=1, \zeta>1$.

When $\gamma=0$, the metric (2.3) now has signature $(-+)$ everywhere except at $x=y=0$. The coordinate transformation (2.4) brings the metric for $x^{2}+y^{2}>0$ again to the form (2.5). If $\varphi$ were not periodic, the transformation

$$
\begin{aligned}
& T=\frac{1}{2}(\zeta-1)^{1 / 2} r \cosh \left(2(\zeta-1)^{-1 / 2} \varphi\right) \\
& X=\frac{1}{2}(\zeta-1)^{1 / 2} r \sinh \left(2(\zeta-1)^{-1 / 2} \varphi\right)
\end{aligned}
$$

would bring this metric into the explicit Minkowski form

$$
d s^{2}=-d T^{2}+d X^{2}
$$

where the range of the coordinates would be $T>|X|$. The periodicity of $\varphi$ therefore means that one has to take the quotient of the domain $T>|X|$ in (2.14) with respect to a boost whose rapidity parameter is $4 \pi(\zeta-1)^{-1 / 2}$. The resulting spacetime is known as (one of) the causal region(s) of Misner space [2]. The full spacetime described by the metric (2.3) with $\gamma=0$ is thus obtained by adding a single point to one causal region of Misner space.

\footnotetext{
${ }^{4}$ Strictly speaking, what we have established is that the $\gamma \rightarrow 0$ limit exists with respect to the topology of pointwise convergence ("weak topology"). For many purposes a finer topology on the space of distributions is desirable, such as that treated in Chapter III of Ref. 48. Convergence with respect to the latter topology actually follows from Theorem XIII of that chapter, but as a rule we will ignore such distinctions herein, as our primary concern is limited to the evaluation of the action integral (1.2).
} 
If the coordinate $T$ is taken to increase towards the future (respectively past), the added point is to the past (future) of every other point, and it can be regarded as analogous to a cosmological initial (final) singularity. Continuing the sartorial imagery of Refs. [37 39], we shall refer to this spacetime as a yarmulke spacetime, and to the critical point at $x=y=0$ as a yarmulke singularity.

We now take the regulator $\gamma$ to be purely imaginary: $\gamma= \pm i \sigma$, where $\sigma>0$. The metric (2.3) is then complex, but everywhere non-degenerate. Writing

$$
\begin{aligned}
M & =\sqrt{\rho^{2}(\zeta-1)-\gamma}, \\
a & =\rho \sqrt{\rho^{2}+\gamma},
\end{aligned}
$$

where $\rho=\sqrt{x^{2}+y^{2}}$ as before, lends the Ricci scalar the form

$$
R=+\frac{2}{a M}\left(\frac{a^{\prime}}{M}\right)^{\prime}
$$

and the volume element is given by

$$
\sqrt{-g} d^{2} x=\rho^{-1} a M d x d y .
$$

Notice that this volume element would have vanished for some positive $\rho$ if we had tried to take $\gamma$ real. We wish to examine the scalar density $\frac{1}{2} \sqrt{-g} R$.

Although the expression (2.16) for $R$ does not depend on the branches chosen for the square roots in (2.15), the expression (2.17) for $\sqrt{-g}$ does. In order to guarantee that the $\gamma \rightarrow 0$ limit of $\sqrt{-g}$ is positive for $\rho>0$, we choose the real parts of the square roots in (2.15) to be positive at large values of $\rho$. Integrating $\frac{1}{2} \sqrt{-g} R$ against a test function $\Phi(x, y)$ and proceeding as in the previous subsection yields then

$$
\frac{1}{2} \int_{\mathbb{R}^{2}} d^{2} x \sqrt{-g} R \Phi=2 \pi\left(\frac{2}{\sqrt{\zeta-1}} \mp i\right) \bar{\Phi}(0)-2 \pi \int_{0}^{\infty} d \rho\left(\frac{a^{\prime}}{M}-\frac{2}{\sqrt{\zeta-1}}\right) \bar{\Phi}^{\prime}(\rho) .
$$

In the limit $\gamma \rightarrow 0$, the integral term on the right hand side of (2.18) vanishes by dominated convergence. Therefore, the $\gamma \rightarrow 0$ limit of $\frac{1}{2} \sqrt{-g} R$ is again a two-dimensional delta-function at $x=y=0$ :

$$
\frac{1}{2} \sqrt{-g} R=2 \pi\left(\frac{2}{\sqrt{\zeta-1}} \mp i\right) \delta_{2}(x, y),
$$

where the sign $\mp$ corresponds to the sign in $\gamma= \pm i \sigma$.

The strength of the delta-function is now complex. Its real part is equal to the rapidity parameter of the Misner space, but there is also an imaginary piece $\mp 2 \pi i$, which is entirely independent of the rapidity parameter. The sign of this imaginary piece depends on the sign of our imaginary regulator $\gamma= \pm i \sigma$. We can fix this sign by requiring that the action

$$
S_{\phi}=-\frac{1}{2} \int \sqrt{-g} d^{2} x g^{a b}\left(\partial_{a} \phi\right)\left(\partial_{b} \phi\right)
$$


for a massless scalar field have a positive imaginary part for real-valued $\phi$ on the regularized spacetime, this being the (formal) condition of convergence for path integrals of the form $\int D \phi \exp \left(i S_{\phi}\right)$ on the regularized spacetime. It is straightforward to verify that satisfaction of this condition is equivalent to the choice $\gamma=+i \sigma$, which yields the upper sign in (2.19):

$$
\frac{1}{2} \sqrt{-g} R=2 \pi\left(\frac{2}{\sqrt{\zeta-1}}-i\right) \delta_{2}(x, y) .
$$

Notice that with the choice $\operatorname{Im} \gamma>0$, the regularized metric satisfies the general condition,

$$
\operatorname{Im}\left(g_{a b}\right)>0
$$

This puts our choice of sign in a broader context, as will be discussed in Section $\square$.

\section{Euclidean double cover conical singularity}

We take now $\epsilon=-1, \zeta<1$.

When $\gamma=0$, the metric (2.3) has signature $(++)$ everywhere except at $x=y=0$. To understand the geometry, we perform the local coordinate transformation

$$
\begin{aligned}
& u=x^{2}-y^{2} \\
& v=2 x y
\end{aligned}
$$

which brings the metric to the explicitly flat form

$$
d s^{2}=\frac{1}{4}(1-\zeta) d u^{2}+\frac{1}{4} d v^{2}
$$

Globally, the transformation (2.23) is the squaring map in the complex plane, $u+i v=$ $(x+i y)^{2}$. This means that for $x^{2}+y^{2}>0$, the metric (2.3) describes the double cover of the punctured flat Euclidean plane. In other words, the metric (2.3) describes a cone with defect angle $-2 \pi$. Note that the defect angle is negative and independent of $\zeta$, so that all the metrics (2.24) describe in some sense the same geometry. Ramifications of this fact will be discussed in Sections $\mathbb{I V}$ and $\nabla \nabla$.

We now take the regulator $\gamma$ to be positive, making the metric everywhere positive definite. Writing

$$
\begin{aligned}
& A=\rho^{2}+\gamma, \\
& B=(1-\zeta) \rho^{2}+\gamma,
\end{aligned}
$$

where $\rho=\sqrt{x^{2}+y^{2}}$ as before, brings the Ricci scalar to the form

$$
R=-2 \gamma A^{-2} B^{-2}\left(2(1-\zeta) \rho^{2}+(2-\zeta) \gamma\right)
$$

and the volume element to the form 


$$
\sqrt{g} d^{2} x=\sqrt{A B} d x d y .
$$

We wish to consider the scalar density $\frac{1}{2} \sqrt{g} R$. Let $\Phi(x, y)$ be a test function. Employing the polar coordinates (2.9), writing $\rho^{2}=r$, and making the definition (2.10), we obtain

$$
\begin{aligned}
\frac{1}{2} \int_{\mathbb{R}^{2}} d^{2} x \sqrt{g} R \Phi & =-\gamma \int_{\mathbb{R}^{2}} d x d y(A B)^{-3 / 2}\left(2(1-\zeta) \rho^{2}+(2-\zeta) \gamma\right) \Phi \\
& =-2 \pi \gamma \int_{0}^{\infty} d \rho \rho(A B)^{-3 / 2}\left(2(1-\zeta) \rho^{2}+(2-\zeta) \gamma\right) \bar{\Phi} \\
& =-\pi \gamma \int_{0}^{\infty} d r(A B)^{-3 / 2}(2(1-\zeta) r+(2-\zeta) \gamma) \Phi \\
& =2 \pi \gamma \int_{0}^{\infty} d r \bar{\Phi} \frac{d}{d r}\left[(A B)^{-1 / 2}\right] \\
& =-2 \pi \bar{\Phi}(0)-2 \pi \int_{0}^{\infty} d r \gamma(A B)^{-1 / 2} \frac{d \bar{\Phi}}{d r},
\end{aligned}
$$

where in the last step we have integrated by parts and used the fact that $(A B)^{1 / 2} \rightarrow \gamma$ as $r \rightarrow 0$. Note that $d \bar{\Phi} / d r$ is finite at $r=0$. In the limit $\gamma \rightarrow 0$, the integral term on the last line of (2.28) vanishes by dominated convergence. Hence, the $\gamma \rightarrow 0$ limit of $\frac{1}{2} \sqrt{g} R$ is a two-dimensional delta-function at $x=y=0$,

$$
\frac{1}{2} \sqrt{g} R=-2 \pi \delta_{2}(x, y),
$$

the strength of the delta-function again being precisely the defect angle. The result therefore agrees with that obtained in subsection $\amalg \mathrm{A}$, whose special case $\zeta=0$ corresponds to the entire family of metrics of the present subsection.

\section{Lorentzian crotch singularity}

Finally we take $\epsilon=-1, \zeta>1$.

When $\gamma=0$, the metric (2.3) has signature $(-+)$ everywhere except at $x=y=0$. The coordinate transformation (2.23) again brings the metric to the form (2.24), which is now explicitly flat Lorentzian. This means that for $x^{2}+y^{2}>0$, the metric (2.3) describes the double cover of punctured two-dimensional Minkowski space. The geometry near the origin is therefore that of the flat $(1+1)$-dimensional trousers spacetime [16,37 41]. To construct a spacetime that was globally a trousers, one would take the star-shaped domain defined by the inequalities

$$
\begin{array}{ll}
|x| y \leq a & \text { for } y \geq 0 \\
|x| y \geq-b & \text { for } y \leq 0
\end{array}
$$

where $a$ and $b$ are positive constants, and on the boundary of this domain one would perform the identifications 


$$
\begin{array}{ll}
(a / y, y) \sim(-a / y, y) & \text { for } y>0, \\
(b / y, y) \sim(-b / y, y) & \text { for } y<0 .
\end{array}
$$

The identified lines become the outer seams of the trousers. The two legs are at $y \rightarrow \infty$ and $y \rightarrow-\infty$, with geodesic circumferences $a$ and $b$ respectively, and the waist is at $|x| \rightarrow \infty$, with geodesic circumference $a+b$.

As in subsection $\mathbb{I I B}$, a real regulator cannot yield an invertible metric. We must therefore again take $\gamma$ to be complex, and again we choose it purely imaginary: $\gamma= \pm i \sigma$, where $\sigma>0$. The metric is then complex, but everywhere non-degenerate. The Ricci scalar is given by (2.26), with $A$ and $B$ as in (2.25), and for the volume element we have now

$$
\sqrt{-g} d^{2} x=\sqrt{-A B} d x d y .
$$

To ensure that the $\gamma \rightarrow 0$ limit of $\sqrt{-g}$ is positive for $\rho>0$, we choose the branch of the square root in (2.32) so that real part of $\sqrt{-g}$ is positive at large values of $\rho$. Integrating $\frac{1}{2} \sqrt{-g} R$ against a test function $\Phi(x, y)$ and proceeding as in the previous subsection yields

$$
\frac{1}{2} \int_{\mathbb{R}^{2}} d^{2} x \sqrt{-g} R \Phi= \pm 2 \pi i \bar{\Phi}(0)+2 \pi \int_{0}^{\infty} d r \gamma(-A B)^{-1 / 2} \frac{d \bar{\Phi}}{d r} .
$$

In the limit $\gamma \rightarrow 0$, the integral term on the right hand side of (2.33) vanishes by dominated convergence. Therefore, the $\gamma \rightarrow 0$ limit of the scalar density $\frac{1}{2} \sqrt{-g} R$ is once again a two-dimensional delta-function at $x=y=0$ :

$$
\frac{1}{2} \sqrt{-g} R= \pm 2 \pi i \delta_{2}(x, y) .
$$

The coefficient of the delta-function is now purely imaginary, and its sign depends on the imaginary part of the regularized metric. Requiring $\operatorname{Im}\left(g_{a b}\right)>0$ for the same reasons as before again fixes $\gamma$ to be positive imaginary, which in turn yields the upper sign in (2.34):

$$
\frac{1}{2} \sqrt{-g} R=2 \pi i \delta_{2}(x, y) .
$$

Notice that the strength of the delta-function is independent of the parameter $\zeta$. As in subsection III, this reflects the fact that all values of $\zeta$ describe, in an appropriate sense, the same geometry. We shall return to this issue in Sections $\mathrm{IV}$ and $\mathrm{VI}$.

\section{ACTION AND CURVATURE FROM THE GAUSS-BONNET THEOREM (OR REGGE CALCULUS)}

We have seen that when our singular metrics are approached within our family of regularized metrics, the Ricci scalar density converges to a delta-function. The strength of this delta-function depends only on the defect angle in the Euclidean case, and only on the rapidity parameter in the Lorentzian case. In this section we recall how the Euclidean results 
follow from the Gauss-Bonnet theorem, and argue that the Lorentzian results can also be recovered from a suitable generalization of the Gauss-Bonnet theorem.

The simplest form of the Gauss-Bonnet theorem states that, given a smooth positive definite metric on a closed two-dimensional manifold, the integral of the scalar density $\frac{1}{2} \sqrt{g} R$ is a topological invariant independent of the metric [49.50]:

$$
\frac{1}{2} \int d^{2} x \sqrt{g} R=2 \pi \chi
$$

where $\chi$ is the Euler number of the manifold. If one requires that this theorem hold also for positive definite metrics with conical singularities, it is a matter of simple geometry to see that the contribution to $\frac{1}{2} \sqrt{g} R$ at the singularity must contain a delta-function whose strength is equal to the defect angle. (For example, for defect angles between 0 and $2 \pi$ one can consider $S^{2}$ with the metric consisting of a cone joined in a $C^{1}$ fashion to a spherical cap that is larger than a hemisphere.) If one further requires that $\frac{1}{2} \sqrt{g} R$ contain at the singularity no worse distributions than the delta-function, the result is then entirely fixed for the curvature, as well as the action. The requirement that there be no derivatives of deltafunctions at the singularity can be motivated by the fact that, for our unregularized metrics, the most singular individual terms in the scalar curvature density diverge like $\left(x^{2}+y^{2}\right)^{-1}$ near the singularity.

The fact that the density $\frac{1}{2} \sqrt{g} R$ is a total divergence in two dimensions remains true for complex-valued metrics. The integral of this density must therefore remain invariant under continuous local deformations of the metric even when the metric is complex. One therefore expects that the Euclidean Gauss-Bonnet theorem can be in some suitable sense analytically continued to complex metrics, and eventually to Lorentzian or almost Lorentzian metrics. For a compact manifold, this would mean continuing (3.1) to

$$
\frac{1}{2} \int d^{2} x \sqrt{-g} R=-2 \pi i \chi .
$$

The sign on the right hand side of (3.2) follows from adopting the usual direction for Wick rotation, or equivalently, from our continuation rule (2.22). Note that (3.2) is in agreement with the fact that a closed two-manifold admits a (strictly) Lorentzian metric only for $\chi=0$; see for example Refs. [18, 19, 51 and the references therein. Note also that a Gauss-Bonnet theorem in two dimensions is known for Lorentzian metrics on compact manifolds with a boundary, when this boundary consists solely of spacelike and timelike segments [52 54]: in this theorem, the left hand side of (3.2) contains also line integral terms from the boundary segments and corner terms from the points where the segments meet.

If (3.2) is assumed to hold for metrics that are almost Lorentzian, our results for the scalar density $\frac{1}{2} \sqrt{-g} R$ at the yarmulke and crotch singularities become immediate, just as in the Euclidean case. To see this for the yarmulke singularity, consider (for example) the manifold $\mathbb{R} \times S^{1}$ with the metric

$$
d s^{2}=-d t^{2}+\sin ^{2}(t) d \theta^{2}
$$


where $0<t<\pi$, and $\theta$ is periodic with period $\beta>0$. Locally (3.3) is just the $(1+1)$ dimensional anti-de Sitter metric with $R=-2$. Adding a point at $t=0$ and another point at $t=\pi$ yields the manifold $S^{2}$ with two yarmulke singularities, each having rapidity parameter $\beta$. The right hand side of (3.2) is equal to $-4 \pi i$, and the contribution to the left hand side from the smooth part is $-2 \beta$ : hence $\frac{1}{2} \sqrt{-g} R$ must have at each singularity a delta-function with the strength $\beta-2 \pi i$, which is the result (2.21).

To obtain the analogous result for the crotch singularity, begin with the trousers spacetime, with the identifications (2.31), and close off the waist and each of the legs with a yarmulke consisting of the half $0 \leq t \leq \frac{1}{2} \pi$ of (3.3) as just described. The result is the manifold $S^{2}$, with one crotch singularity and three yarmulke singularities. The nonvanishing volume integrals are essentially the same as before, and with the result for the yarmulke singularity known, (3.2) immediately yields for $\frac{1}{2} \sqrt{-g} R$ at the crotch singularity the result (2.35). Alternatively, instead of closing off the legs and waist, one can employ the Gauss-Bonnet theorem for manifolds with boundary [55]; the result is the same.

The subtlety with the anticipated complex Gauss-Bonnet theorem is that when the metric is complex, the invariance of $\frac{1}{2} \int d^{2} x \sqrt{g} R$ under continuous deformations of the metric is not by itself sufficient to fix the value of the integral of this density in terms of the topology of the manifold: there exist complex metrics for which neither (3.1) nor (3.2) holds, even after taking into account the possibility of the global sign ambiguity in $\sqrt{g}$. Nevertheless, it appears plausible that one can formulate a complex Gauss-Bonnet theorem by placing suitable restrictions of a topological nature on the complex metric involved. These restrictions could then be used to specify a class of regularizations within which the resulting value for the Ricci scalar density would agree with ours - something like regularizations for which the complex metric has positive imaginary part and contains some positive definite metric in its connected component. One might also expect to be able to characterize the appropriate connected component by a "winding number" of the cross-section it represents of the bundle of complex-valued metrics on the manifold. We have not attempted to explore this question in a systematic fashion, however.

\footnotetext{
${ }^{5}$ For example, $S^{2}$ admits smooth invertible complex metrics for which $\frac{1}{2} \int_{S^{2}} d^{2} x \sqrt{g} R=0$, whereas for positive definite metrics the Gauss-Bonnet theorem (3.1) implies $\frac{1}{2} \int_{S^{2}} d^{2} x \sqrt{g} R=4 \pi$. An explicit example follows. Define the function $f:(0, \pi) \rightarrow \mathbb{C}$ by $f(\tau)=\sin (\tau)\left[1+i \sin ^{2}(\tau) \cos (\tau)\right]$. Consider the metric $d s^{2}=\left[f^{\prime}(\tau)\right]^{2} d \tau^{2}+f^{2}(\tau) d \varphi^{2}$, where $f^{\prime}=d f / d \tau$ and $\varphi$ is periodic with period $2 \pi$. Near $\tau=0$ and $\tau=\pi$ one can introduce new local coordinates (for example, $x=\tau \cos \varphi$ and $y=\tau \sin \varphi$ near $\tau=0$ ), in which one sees that $\tau=0$ and $\tau=\pi$ are just coordinate singularities and that the metric can be naturally completed into a nondegenerate metric on $S^{2}$. As the metric is locally related by a complex diffeomorphism [56] to the flat Euclidean metric in polar coordinates, $d s^{2}=d r^{2}+r^{2} d \varphi^{2}$, the Riemann tensor vanishes, and hence $\frac{1}{2} \int_{S^{2}} d^{2} x \sqrt{g} R=0$. (The total volume of this metric vanishes, $\int_{S^{2}} d^{2} x \sqrt{g}=0$, but one can easily deform the metric locally in a $C^{\infty}$ fashion to make the volume nonzero while retaining $\frac{1}{2} \int_{S^{2}} d^{2} x \sqrt{g} R=0$.)
} 
Finally, we note that the complex angles that implicitly occur in Lorentzian Regge calculus provide yet another route to our basic results for the integrated scalar curvature. If one subdivides a neighborhood of the crotch singularity (say) into flat simplicial blocks, then the defect angle can be computed easily using the "complex trigonometry" of the appendix of Ref. [20]. The answer again agrees with what we have found above, provided one resolves the complex-conjugation ambiguity analogously.

\section{TENSOR DENSITIES AND DISTRIBUTIONAL CURVATURE}

Until now we have concentrated on the Ricci scalar density. In this section we shall briefly comment on the possibilities for giving a distributional interpretation to other curvature quantities of interest.

At a general level, we recall that the ordinary delta-function on a manifold can, by definition, be integrated against a test scalar without invoking a volume element: in a local coordinate system $\left(x^{1}, x^{2}, \ldots, x^{n}\right)$ whose origin is at the point of support of the deltafunction, the single component of the ordinary delta-function is just $\delta\left(x^{1}\right) \delta\left(x^{2}\right) \cdots \delta\left(x^{n}\right)$. This means that the ordinary delta-function should be viewed as a singular scalar density of weight one, not as a singular function of weight zero. Indeed, as the ordinary delta-function is a measure, it requires only $C^{0}$ test functions, and hence it is insensitive to the choice of the differentiable structure (depending only on the manifold's topology). In contrast, the scalar (or "covariant") delta-function $( \pm g)^{-1 / 2} \delta$, defined on a (pseudo-)Riemannian manifold, can be thought of as a singular function of weight zero: densities of weight one, and the definition of such test densities requires knowledge of the differentiable structure.

Now, we have seen that the Ricci scalar density for our singular metrics is proportional to the ordinary delta-function on the two-manifold. Although we worked within a particular set of coordinates, both sides of this equation are singular densities of weight one, and the equation therefore must hold in arbitrary coordinates, and with any choice of the differentiable structure. In effect, we already relied on these properties in Section III when using the Gauss-Bonnet theorem to show that the result is independent of the details of the regularization. What we wish to emphasize here is that the result is not tied to the differentiable structure defined by our coordinate chart $(x, y)$.

The reason for making the above point is that the differentiable structure defined by the chart $(x, y)$ need not always be a natural one from the viewpoint of the $\gamma=0$ geometry.

\footnotetext{
${ }^{6}$ On a manifold that has not been endowed with a volume element it is not possible to define a unique delta-function of density weight zero. The best one can do is define a one-parameter family of such delta-functions, all of which are scalar multiples of each other, but a unique choice of normalization requires a volume element.
} 
For example, when $\epsilon=1$ and $\zeta=-3$, the defect angle vanishes and the metric (2.3) can be thought of as describing a plane, but the usual Cartesian coordinates on the plane are then not differentiable functions in the chart $(x, y)$ at the origin. For $\epsilon=-1$, all values of $\zeta<1$ give a metric with the defect angle $-2 \pi$, but if one seriously interprets the metric (2.3) with $\gamma=0$ and two different values of $\zeta<1$ as describing the same geometry in two different coordinate systems, one finds that the transformation between the two coordinate systems is continuous but not differentiable at the origin. Completely analogously, for $\epsilon=-1$, all values of $\zeta>1$ give a metric with "the same" crotch singularity at the origin, but if one interprets the metric (2.3) with $\gamma=0$ and two different values of $\zeta>1$ as describing the same geometry in two different coordinate systems, the coordinate transformation is not differentiable at the origin. None of this raises concerns about our result for the Ricci scalar density, however. Indeed, for $\epsilon=-1$, the result obtained in Section $\amalg$ was explicitly seen to depended on $\zeta$ only through whether $\zeta<1$ or $\zeta>1$.

If one attempts to derive from our metrics distributional curvature tensors (or scalar densities of weight other than one), the role of the differentiable structure becomes more important. One might still expect the Riemann and Ricci curvatures to be concentrated at the singular point as some sort of delta-function, but any such relation would now have to rely on the specification of a reference differentiable structure, or at least a reference volume element. This relative character of the curvature being defined would make it more difficult to ascertain to what extent its distributional limit depended on the particular regularization chosen.

As an example, consider the Riemann tensor for our metrics. When the metric is invertible, the independent components of the Riemann tensor $R_{b c d}^{a}$ in the chart $(x, y)$ take the form

$$
\begin{aligned}
& R_{x x y}^{x}=\frac{1}{2} g_{x y} R, \\
& R_{y x y}^{x}=\frac{1}{2} g_{y y} R, \\
& R_{x x y}^{y}=-\frac{1}{2} g_{x x} R, \\
& R_{y x y}^{y}=-\frac{1}{2} g_{x y} R .
\end{aligned}
$$

With $\gamma$ chosen as in the previous section, positive for $\zeta<1$ and positive imaginary for $\zeta>1$, it is straightforward to integrate the expressions (4.1) against a test function and to take the limit $\gamma \rightarrow 0$. For $\zeta<1$, the resulting nonvanishing independent components of $R_{b c d}^{a}$ are given by

$$
\begin{gathered}
\epsilon=1: \quad R_{y x y}^{x}=-R_{x x y}^{y}=\pi\left[\frac{-1}{(1-\zeta)}+\frac{(2+\zeta) \ln (1-\zeta)}{2 \zeta}\right] \delta_{2}(x, y), \\
\epsilon=-1: \quad R_{y x y}^{x}=-R_{x x y}^{y}=\pi\left[-1+\frac{(2-\zeta) \ln (1-\zeta)}{2 \zeta}\right] \delta_{2}(x, y)
\end{gathered}
$$

where at $\zeta=0$ the expressions are to be understood in the sense of the limit $\zeta \rightarrow 0$. For $\zeta>1$, the results are obtained from those in (4.2) through replacing $\ln (1-\zeta)$ by $\ln (\zeta-1)+i \pi$. 
Thus we can say that our Riemann tensor, regarded as a distribution acting on weight one test fields $\Phi_{a}{ }^{b c d}$ that are smooth in our differentiable structure, has a well-defined $\gamma \rightarrow 0$ limit for each value of the parameters $\zeta$ and $\epsilon$. However, these limits have highly unsatisfactory properties. For example, (4.22) does not vanish for $\zeta=-3$, even though the defect angle then vanishes and the cone reduces to a plane. Instead, (4.2a) vanishes for precisely two values of $\zeta$, one corresponding to a positive and the other to a negative defect angle. Similarly, (4.2b) vanishes for precisely one value of $\zeta<1$, even though the metric has a defect angle equal to $-2 \pi$ for all values of $\zeta<1$. This highlights the difficulties discussed in Ref. 四 for defining a distributional Riemann tensor for conically singular metrics: the distributional limit is highly sensitive to the choice of the regularization, and specifically to the choice of differentiable structure that the regularization implicitly uses.

As a second example, consider the Ricci scalar $R$. As the Ricci scalar density for our two-dimensional singular metrics is proportional to the ordinary delta-function $\delta_{2}$, one might attempt to define the Ricci scalar $R$ as a distribution proportional to the scalar delta-function $( \pm g)^{-1 / 2} \delta_{2}$. The problem with this is that for our metrics the scalar delta-function is not defined, because the factor $( \pm g)^{-1 / 2}$ is singular. Note, however, that for the Euclidean conical singularity of subsection $\llbracket \mathrm{A}$, the Ricci scalar can be defined as a distribution proportional to the delta-function if one changes the differentiable structure from that defined by the coordinate functions $(x, y)$ to that defined by the functions $(\xi, \eta)$, where [57]

$$
\begin{aligned}
& \xi=x \sqrt{x^{2}+y^{2}}, \\
& \eta=y \sqrt{x^{2}+y^{2}} .
\end{aligned}
$$

The transformation (4.3) is smooth everywhere except at the origin, where it is only $C^{0}$ and its Jacobian diverges. One can view this singular Jacobian as canceling the singularity that occurred in the factor $(g)^{-1 / 2}$ in the coordinates $(x, y)$.

\section{PHASE OF THE COMPLEX REGULATOR}

For the yarmulke singularity and the crotch singularity, the sign of the imaginary part of the Ricci scalar density was fixed by the choice that the regulator $\gamma$ have a positive imaginary part. In this section we shall discuss the status of this choice.

In Section [1] we deduced the sign of the imaginary part of $\gamma$ from the condition (2.22), which itself was chosen to give a positive imaginary part to the action (2.20) of a real massless scalar field. Including a mass term in the action (2.20) would not have made a difference for

\footnotetext{
${ }^{7}$ At first sight it might seem paradoxical that the scalar $R$ should be more sensitive to the choice of differentiable structure than the density $\sqrt{g} R$. However, this impression disappears if one remembers that a distribution is essentially a dual object, and dualization reverses the roles of scalars and scalar densities.
} 
$1<\zeta \leq 2$. For $\zeta>2$, however, the imaginary parts of the mass term and the kinetic term in the action have the opposite sign, and the scalar field path integral on the regularized spacetime is not convergent for either sign of the regulator. The same difference between the cases $1<\zeta \leq 2$ and $\zeta>2$ occurs also for the Maxwell field on a spacetime that is a product of one of our $(1+1)$-dimensional metrics with two flat Euclidean dimensions.

One solution to the problem is to rotate the metric parameter $\zeta$ into the complex along with $\gamma$. In fact, the ansatz $\gamma=i \sigma+\eta, \zeta=\zeta_{0}-i \eta$, with $\eta=\sqrt{\left(\zeta_{0}-2\right) \sigma}, \zeta_{0}>2$, takes care of both the electromagnetic and scalar fields, and with it one still obtains the same limiting values (2.21) and (2.35) for $\frac{1}{2} \sqrt{-g} R$ as $\gamma \rightarrow 0$. As far as the scalar field alone is concerned, the difficulty could also be cured by rotating the coupling constant $m^{2}$ into the complex, but such a technique would not help with the Maxwell field, or with nonabelian gauge fields.

This construction of a regulator may be satisfactory as far as it goes, but, without further motivation, it seems rather ad hoc, and it doesn't clearly guarantee that an entirely different choice of regulator might not lead to a very different outcome. A more systematic approach would replace (2.22) (or, slightly more generally, the condition $\operatorname{Im}\left(g_{a b}\right) \geq 0$ ) by a condition that would guarantee convergence for any reasonable matter field. (Linearized gravity is also important, of course, but is something of a special case [58.) It turns out that if we restrict ourselves to local conditions on small deformations $\delta g_{a b}$ away from non-degenerate Lorentzian metrics, then an optimum condition is that the deformation be of the form

$$
\delta g_{a b}=i \sum_{\epsilon} \epsilon_{a} \epsilon_{b}
$$

where each covector $\epsilon_{a}$ is real and timelike (or null) with respect to $g_{a b}$. This will guarantee (formal) convergence of the path integral for any field whose classical stress-energy tensor satisfies (off shell) the weak energy condition $\left(T^{a b} v_{a} v_{b} \geq 0, \forall\right.$ timelike $\left.v_{a}\right)$. Evidently (5.1) implies (2.22), but is more restrictive. For finite deformations, there exist analogous strengthenings of (2.22) that work for individual matter fields but they are harder to state. 8 We have not attempted to generalize the infinitesimal condition (5.1) or its finite analogues

\footnotetext{
${ }^{8}$ It follows from (2.22) that one can find a basis of (real) vectors in which $g_{a b}$ is diagonal, i.e., in which it assumes the form $g_{a b}=\sum_{j} \lambda_{j}\left(v_{j}\right)_{a}\left(v_{j}\right)_{b}$. The strengthenings referred to can then be expressed as conditions on the $\lambda_{j}$. For the massless scalar field, we want $\operatorname{Im}\left(\sqrt{-g} g^{a b}\right)<0$ and hence need $\operatorname{Im}\left(\sqrt{-\prod \lambda} / \lambda_{j}\right)<0$ for all $j$; with a mass term present, we need also $\operatorname{Im} \sqrt{-\prod \lambda}<0$. For the electromagnetic field the condition is positivity of the imaginary part of the quadratic form $Q(F):=-(1 / 4) \sqrt{-g} g^{a b} g^{c d} F_{a c} F_{b d}$, corresponding to the requirement $\operatorname{Im}\left(\sqrt{-\prod \lambda} /\left(\lambda_{j} \lambda_{k}\right)\right)<0$ for all $j \neq k$. Here, $g_{a b}$ must be in the same connected component (with respect to the above conditions) as some real metric of Lorentzian signature, and the branch of the square root is chosen to be positive for that metric. It is easy to verify that all of these conditions are satisfied along the path of ordinary Wick rotation, which turns $g_{\mu \nu}=\operatorname{diag}(-1,1,1 \ldots .1)$ into $g_{\mu \nu}=\operatorname{diag}(1,1,1 \ldots .1)$ by taking $g_{00}$ from -1 to +1 through the upper half plane. We know of no general condition analogous to (5.1) for finite deformations that is guaranteed to cover all possible matter fields.
} 
to regions where the metric degenerates, such as at yarmulke or crotch singularities, but the existence of the ansatz written down in the previous paragraph strongly suggests this should be possible.

Even without such a generalization in hand, it seems sufficiently clear that any adequate regulator must lead to the same values as found in Section $\mathbb{\text { II }}$ for the Ricci scalar density. Indeed, we obtained these values by temporarily deforming the metric into the space "CI" of complex invertible metrics, and the discussion of Section III shows that the only possible ambiguity associated with this procedure arises from the choice of which connected component of $C I$ one deforms into. But the weakest of the convergence conditions we have entertained, namely (2.22), already defines a domain $D \subseteq C I$ that is convex, and therefore connected. Hence the Gauss-Bonnet integral must remain constant within $D$, and no ambiguity can arise. (For consistency $\sqrt{-g}$ must be single-valued within $D$, which in fact it is, thanks to the convexity of D.) Clearly, no further conditions imposed in addition to (2.22) can affect this conclusion. In particular, any regulator respecting (2.22) will resolve the sign ambiguity in (2.19) and (2.34) in the same way as led to our basic results, (2.21) and (2.35). Essentially the same argument for uniqueness can also be made in the context of Regge calculus, where the sign of the imaginary part of the action hinges on how one resolves the complex conjugation ambiguity in the definition of a Lorentzian angle (cf. [20]). Here again, an analytic continuation along a path of metrics respecting the condition (2.22) suffices to fix the sign uniquely, and one obtains contributions to the action entirely consistent with (2.21) and (2.35), namely $\beta-2 \pi i$ for the yarmulke and $\beta+2 \pi i$ for the trousers.'

Further insight into the inevitability of (2.21) and (2.35) comes from considering their dependence on the parameter $\zeta$. The requirement that a scalar field path integral on the regularized Lorentzian geometry should be formally convergent is closely connected with the reasons that mandate the direction of the Wick rotation in flat-space quantum field theory. Thus, we might expect that our results for the crotch and yarmulke singularities could also be derived from the analogous formulas for conical singularities of Euclidean signature by viewing the Euclidean and Lorentzian Ricci scalar densities as analytic continuations of each other in the parameter $\zeta$. To see that this is so, recall that the usual direction of Wick rotation, as specified for example by (2.22), implies that the Euclidean expression $\sqrt{g} R$ continues to the Lorentzian expression $+i \sqrt{-g} R$. [The relation $i S=-S_{\mathrm{E}}$ then leads to the usual definition

$$
S_{\mathrm{E}}=-\frac{1}{2 \kappa} \int d^{D} x \sqrt{g} R+\text { (boundary terms) }
$$

for the Euclidean counterpart of the Einstein action ([1.2]).] This same rule that the Euclidean

\footnotetext{
${ }^{9}$ The second expression refers to a generalized crotch singularity, obtained from that of Section [I] by removing a wedge of rapidity parameter $\beta$. The sign of $\beta$ is positive (respectively negative) if the wedge points in a spacelike (timelike) direction.
} 
$\sqrt{g}$ continues to the Lorentzian $i \sqrt{-g}$ is embodied in the formulas given in Section $\mathbb{1}$ for $\sqrt{g}$ and $\sqrt{-g}$, as one sees by setting $\gamma$ to zero, and analytically continuing $\zeta$ past $\zeta=1$ in the lower half plane (this choice of half-plane being the one implied by the condition $\operatorname{Im}\left(g_{a b}\right) \geq 0$ ). Comparing, then, (2.29) with $i$ times (2.35), we see that the crotch curvature density is indeed the analytic continuation of its counterpart for the Euclidean double cover metric. The $\epsilon=1$ case is similar, but slightly more interesting, because of the nontrivial $\zeta$ dependence of the formulas (2.12) and (2.21). Once again we see that $i$ times $\frac{1}{2} \sqrt{-g} R$ of the yarmulke and $\frac{1}{2} \sqrt{g} R$ of the Euclidean general conical singularity are analytic continuations of each other. For the signs of the $\zeta$-dependent terms to agree, $\zeta$ must be continued past $\zeta=1$ in the lower half plane, consistently with what we just observed in connection with $\sqrt{g}$. One can think of the Euclidean defect angle being continued to the complex value $2 \pi+i \beta$, where $\beta>0$ is the rapidity parameter of the Misner space. Equivalently, one can say that the Euclidean opening angle is continued to the purely imaginary value $-i \beta$.

\section{CONCLUSIONS AND DISCUSSION}

In this paper we have investigated two one-parameter families of $(1+1)$-dimensional topology-changing metrics that contain Lorentzian analogues of conical singularities. For the metrics of the first family, the spacetime is a "yarmulke" obtained by adding an initial (or final) singularity to one causal domain of a Misner space. For the metrics of the second family, the singularity is that occurring at the crotch of the Lorentzian "trousers" spacetime. Regularizing the metrics by adding a small positive imaginary part and then taking this regulator to zero, we found that in both cases the scalar density $\frac{1}{2} \sqrt{-g} R$ converges to a delta-function at the singularity. For the trousers family the coefficient of the delta-function is the purely imaginary number $+2 \pi i$, independently of the parameter; for the yarmulke family it is the complex number $\beta-2 \pi i$, where $\beta$ is the rapidity parameter of the associated Misner space.

In these coefficients, the signs of the imaginary parts follow from our having chosen the imaginary part of the regularized metric to be positive. This property guarantees in particular that a scalar field functional integral on the regularized spacetime is formally convergent.

For certain ranges of the parameter $\zeta$ in (2.3), the metrics of our two families acquire a Euclidean signature, and the singularity is then of the ordinary conical sort. In these cases, we verified that our regularization method, with the regulator chosen real, renders the scalar density $\frac{1}{2} \sqrt{g} R$ as a delta-function concentrated at the singularity, with a strength precisely equal to the defect angle. (Curiously, this angle is independent of $\zeta$ for the metrics of the "trousers" family.) This is the familiar result that makes the Gauss-Bonnet theorem hold for Euclidean signature metrics with conical singularities.

In all cases, both Riemannian and Lorentzian, our results are consistent with the general rule that the contribution to the action integral (1.2) from a conical-type singularity equals 
the generalized defect angle, as defined by the "complex trigonometry" natural to Regge calculus [20]. This is also what one would obtain from a suitably complexified GaussBonnet theorem, as discussed in Section IIII. We did not investigate a crotch singularity with nonzero rapidity parameter $\beta$, because such a geometry does not occur among the Morse theory-inspired families of metrics we considered. However, the results of Sections [I] and $\Pi 11$ strongly suggest that we would obtain a delta-function with strength $\beta+2 \pi i$ in that case as well.

The main interest of our findings lies in their implications for topology change. The first issue that arises in this context is whether topological transitions can proceed as classical processes in the classical limit of quantum general relativity. In $D \geq 3$ spacetime dimensions, Einstein gravity in the metric formulation can be defined in terms of a variational principle with the action functional (1.2). Under the usual smoothness and invertibility assumptions for the metric, stationarity of the action under appropriate boundary conditions is equivalent to the vacuum Einstein equations, $G_{a b}=0$. However, stationarity of the action $S$ can be regarded, more generally, as a criterion for selecting classical solutions among all field configurations for which $S$ is defined and suitably differentiable, even when the metrics contain singularities that make the interpretation of the Einstein equations as such problematic. T囗

In this sense, metrics containing Euclidean conical singularities are known not to be vacuum solutions to the metric formulation of Einstein gravity [6.33 [35], the reason being in essence that the contribution to $S$ from the vicinity of such conical singularities is proportional to the defect angle in the 2-dimensional submanifold times the $(D-2)$-dimensional volume of the remaining dimensions, and this contribution is not stationary under variations of the $(D-2)$-dimensional volume unless the defect angle vanishes; for details, see Refs. [6]33] [35]. The results of this paper indicate that a similar conclusion holds for spacetimes that contain Lorentzian singularities of the type we have investigated. For example, the product of one of our singular 2-metrics with a flat torus of $D-2$ dimensions cannot be regarded as a solution of Einstein gravity in the metric formulation.

\footnotetext{
${ }^{10}$ This reasoning assumes that the field equations of classical gravity emerge from a quantum action functional in the same way that Maxwell's equations emerge from the action of the corresponding quantum theory, and not, for example, in the way the heat equation emerges from the path integral for the individual molecules. It is possible that this assumption is too simple, but if so one would have to explain why the classical theory has any variational formulation at all.

${ }^{11}$ Specifically, the action $S$ of such a product is not stationary under certain variations that rescale the metric of the torus by a scaling function of compact support. In fact, if we choose the scaling function to be constant in a neighborhood of the singularity, then the spacetime retains its product form in that neighborhood (globally it is a "warped product") and the Ricci scalar density remains a delta-function there, making it easy to compute the overall action. No doubt there are
} 
If topological transitions thus are forbidden in the classical limit, the next question that arises is whether they can proceed as quantum tunneling processes. Let us consider this question from the vantage point of path-integral quantization. As our product metrics are not stationary points of the action $S$ in $D \geq 3$ dimensions, one would not expect the path integral to gain an appreciable contribution from them. However, the situation is complicated by the fact that $S$ is now complex. On one hand this means that destructive interference will be associated only with non-stationarity of the real part of $S$. (Non-stationarity of $\operatorname{Im}(S)$ entails no suppression as such, although it does imply the presence of nearby paths with amplitudes of greater absolute magnitude.) On the other hand, the very existence of an imaginary part can now lead to its own suppression or enhancement. Indeed the action (1.2) has an imaginary part proportional to the $(D-2)$-dimensional volume of the singularity, and this $(D-2)$-dimensional volume may be large. For the crotch singularity the imaginary part of the action is positive, and the contribution to the path integral from the trousers spacetimes is therefore suppressed by an exponential factor. ${ }^{\text {T2 }}$ Topology change via the trousers mechanism is therefore very strongly disfavored, the suppression factor in $3+1$ dimensions being $\exp (-A / 4 G)$, where $A$ is the area of the two flat dimensions. For the yarmulke singularity, in contrast, the imaginary part of the action is negative, and the contribution to the path integral from the yarmulke spacetimes is therefore enhanced by an exponential factor. One may perhaps take this as evidence for an exponential enhancement

many more (and more localized) variations under which $\delta S \neq 0$, but to render such a statement meaningful one would first have to extend the analysis performed in this paper to define $S$ for the more general class of conically-singular metrics to which such variations would lead.

At a very technical level, there arises in this connection the issue of how even to define a differentiable structure for the enlarged class of metrics under consideration. (This issue would become entangled with that of asymptotic boundary conditions, were one to allow the conical type singularities to extend out to infinity, a point emphasized to us by Abhay Ashtekar.) However, it may be premature to try to resolve such technical issues at this point. Does one even expect the relevant space of generalized metrics to form a smooth manifold for example? The criterion for calling a geometry a classical solution should, in our view, ultimately be chosen to express the condition that the paths in its neighborhood make a non-negligible contribution to the gravitational functional integral, but the precise meaning of this criterion, not to mention its precise relation to attributes such as differentiability of the classical action, is far from settled at this point, all the more so if a fundamental discreteness proves to be required before the gravitational functional integral can be given precise meaning. For a discussion of issues related to the above, see Refs. [6, 32 [35, 59, 60].

${ }^{12}$ There is an obvious, but potentially misleading, analogy here with tunneling solutions, whose complex action also entails suppression. In that case, however, the history or path is itself complex and therefore without direct physical meaning, being of interest only as a saddle point of the analytically extended amplitude. In contrast, the paths under consideration in this paper represent possible histories of the actual gravitational field, even if they are not extrema of $S$. 
of the creation (or annihilation) of a universe by such a mechanism. [3] However, one would need to balance this enhancement against the suppression coming (when $D \geq 3$ ) from the fact that (unlike for the trousers) the yarmulke spacetimes have a Ricci scalar density with non-zero real part, and therefore are not stationary points of $\operatorname{Re}(S)$.

A second implication of our results concerns two dimensional spacetimes as such, where the effect of the action being complex is still present, even if it is not exponentially large. In two dimensions, the relative enhancements and suppressions attaching to the yarmulke and trousers are strongly reminiscent of the topological expansion in string theory. However, in that situation there seems to be no strong evidence about the sign of the coupling constant $\kappa$, unlike in $(3+1)$-gravity, where not only stability, but experiment dictates that $\kappa$ be positive. In any case, it seems worthwhile to ask whether the findings of this paper have any significance for strings.

Our results for the metric formulation provide a striking contrast to the vielbein-cumconnection formulation of Einstein gravity, where a spacetime obtained as the product of one or more flat dimensions with (for example) the double cover conical singularity or the crotch singularity can be regarded as a solution to the classical field equations [41]. Formally, this difference stems from the different roles played on the one hand by the Christoffel connection in the metric formulation, and on the other hand by the Lorentz connection in the vielbein-cum-connection formulation. Our metric action for singular metrics was defined in terms of a limiting process through nonsingular metrics. Since the Christoffel connection is uniquely determined by the metric regardless of whether the field equations hold, the (possibly singular) limiting behavior of the Christoffel connection upon approaching our singular metrics is uniquely determined by the limiting process. In the connection formulation, on the other hand, the relation between the Lorentz connection and the vielbein is an equation of motion, and this equation is well defined even when the vielbein is degenerate. One can therefore directly look for solutions to the full field equations containing singular vielbeins, without having to interpret the latter by reference to a regularized vielbein field. We shall illustrate this in Appendix A for a $(2+1)$-dimensional spacetime that is the product of the Euclidean double cover conical singularity with a time dimension 41, and in Appendix B for the Lorentzian "hourglass" spacetime 42,43. However, this formal analysis of why the two formulations differ leaves open the physical meaning of the difference. In the metric formulation the trousers does not provide a classical route to topology change, whereas in the vielbein-cum-connection formulation, it apparently does. How can these conclusions be reconciled?

\footnotetext{
${ }^{13}$ Is there any useful analogy between this exponentially large factor and the similar factor in the wave function that Hartle and Hawking obtained from the no-boundary path integral in the positive curvature Friedmann model with a cosmological constant 61 63]? Likewise, is there more than a formal significance in the fact that the coefficient of the area that enters here $(8 \pi / \kappa=1 / 4 G)$ is precisely the same one that occurs in computing the entropy of a black hole horizon (cf. [33, 34, 64])?
} 
There would seem to be at least three possible answers to this question. First, it might be that, properly understood, the conditions for a configuration to represent a possible classical evolution are not actually satisfied by the putative solutions, even in the vielbeincum-connection version of Einstein gravity. This might occur, for example, through some subtle failure of the putative solution to possess a neighborhood of sufficient functional measure, but we have not been able to find any convincing reason why that should be the case. (However, the situation appears to be subtle; compare the discussion of " $C^{0}$ isometries" below.) A second possibility is that the trousers is a solution, but that it belongs to an entirely disjoint sector of history space, such that the vielbein-cum-connection formulation agrees with the metric formulation when these sectors are excluded by hand. The third possibility is that the two formulations are irredeemably different, with one allowing classical topology change and the other forbidding it. In either of the last two cases, one must decide which formulation is physically correct, and here it seems the experimental evidence strongly favors the metric formulation. Otherwise, we would have no apparent explanation for why the macroscopic topology of spacetime is not incessantly and prolifically changing in a radically unpredictable manner [41]. (Assuming such changes could proceed with anything like a classical amplitude, the shear variety of possibilities would seem to make their occurrence inevitable on entropic grounds.) If borne out, this conclusion would present one of the rare instances where one can experimentally decide between contending theories of quantum gravity.

Taken as a whole, the findings reported here provide convincing, if indirect, evidence in favor of our complex expressions for the integrated scalar curvature. What is lacking is a deeper understanding of the origin of the imaginary part of these integrals, and more generally of the significance of the complex regularizations we have employed. To provide a straightforward meaning to our regularized action in the context of the Lorentzian sumover-histories, one would have to first define a purely Lorentzian path integral incorporating non-invertible metrics of the type we have been considering, and then show that a conicaltype singularity in the metric introduces an extra suppression or enhancement factor, either because the amplitude itself is not of unit modulus or (to the extent that the distinction makes sense) because the measure factor is changed by the presence of the singularity. We suspect that such a demonstration is not fully feasible within a continuum theory, because the path integral itself is ultimately not definable there, but that something of the sort could emerge from a theory with a built in discreteness, such as provided by a causal set account of spacetime.

In this connection, it seems natural to look for some relationship between our complex action and the divergent stress-energy tensor induced by a crotch or yarmulke singularity via its influence on the quantum matter fields (or gravitons) in its neighborhood [16, 37 41]. The suppression of topology change one would infer from these radiative effects seems reminiscent of the exponential suppression discussed above coming from $\operatorname{Im}(S)$ (but what of the enhancement in the yarmulke case?). Similarly, such radiative effects might help resolve the 
discrepancy discussed earlier between the metric formulation of quantum gravity and the vielbein-cum-connection formulation.

Finally, we would like to raise two general questions that are suggested by the work described above.

The first question is one of principle touching the meaning of "general covariance" in the context of non-invertible metrics such as we have been concerned with. Within the four families of spacetimes we have been working with, there are many examples of pairs of metrics that represent the "same" geometry (and share the same action and distributional scalar curvature density), but nevertheless are not isometric via any diffeomorphism. For example, the metric of subsection $\llbracket \mathrm{A}$ becomes a cone with zero defect angle when $\zeta=-3$, but, as discussed in Section IV, there is no smooth and smoothly invertible transformation of the coordinates $x$ and $y$ that would bring this metric to an explicitly flat Euclidean form (cf. equation (2.4)). Similarly, all of the metrics of the "trousers" family of subsection IID are characterized by a conical singularity of the same strength, yet again there exists no diffeomorphism taking one value of $\zeta$ to another. In the context of invertible metrics, we are used to defining a "geometry" as a diffeomorphism equivalence class of metrics, and declaring that any two metrics that define the same geometry should be identified physically ("general covariance"). But if we want to make a physical identification among all of our trousers metrics, or among all of our double cover conical singularity metrics, then a broader notion of equivalence must be adopted. To do otherwise would lead to a multiple counting of such "histories" in the path integral, which presumably would be incorrect.

Thus we arrive at the view that the class of valid "gauge transformations" is not exhausted by the diffeomorphisms. Rather a more general subset (seemingly not a subgroup!) of the homeomorphism group seems to be indicated, resulting in a more general notion of equivalence that might be termed "generalized (or $C^{0}$ ) isometry". Said another way, this means that the same geometry can be given more than one differentiable structure, without any genuine physical change having occurred. This may be seen as suggesting that the differentiable structure has in some ways less "reality", than, say, the topology or the metric itself. But how, precisely, should one define the concept of generalized isometry? In a Riemannian setting, there is apparently no trouble, since one can just define a generalized isometry to be any homeomorphism preserving the global distance function, which remains well defined even in the presence of singular points where the metric degenerates or vanishes. In the Lorentzian setting, on the other hand, it is not so obvious how to proceed. Plausibly, the appropriate definition is that a generalized isometry is a homeomorphism $f$ that preserves both the local causal order and the volume element $\sqrt{-g}$ (cf. 665). These requirements are meaningful for the type of metric we studied in Section $\mathbb{1 1}$, and they offer a natural generalization of the usual definition, since they can be shown to imply that $f$ is an isometry in the ordinary sense when both metrics involved are smooth and invertible.

Finally, we may ask to what extent the complex actions we have found are characteristic features of topology-changing spacetimes in general, and to what extent they are peculiar 
to two dimensions and the particular family of metrics we have chosen to study. Concerning dimensionality, we may observe that the scalar curvature of metrics like those of Section [I] diverges no more strongly in higher dimensions than it does in two. But this means that the Ricci scalar density is less singular, so that one might anticipate less of a need for any sort of regularization in order that the Hilbert action $S$ be defined. We might thus expect that the occurrence of an imaginary part of this $S$ is indeed peculiar to $(1+1)$-dimensional spacetimes. On the other hand, we may also anticipate that within two dimensions, a complex value for $S$ is an unavoidable concomitant of topology change. This expectation derives from the observation that topology change entails a modification of the light cone structure, and this change can be interpreted in terms of a complex defect angle in the sense discussed in Section III. And because of the generality of the considerations of that section, one would expect a corresponding generality in the conclusion that a complex defect angle implies a complex action. (Similarly, we would expect the Lovelock actions 66,67 to acquire well-defined imaginary parts as a result of topological transitions in higher dimensions.)

\section{ACKNOWLEDGMENTS}

We would like to thank Arley Anderson, Abhay Ashtekar, Luca Bombelli, Arvind Borde, Tevian Dray, John Friedman, Dmitri Fursaev, Domenico Giulini, Gary Horowitz, Ted Jacobson, Klaus Kirsten, Jean Krisch, Don Marolf, and Don Page for useful discussions and correspondence. We would also like to thank Paul Ehrlich, Steve Harris, and Adam Helfer for bringing Refs. [52 54 to our attention. This work was supported in part by the NSF grants PHY-90-16733, PHY-91-05935, PHY-93-07570, PHY-94-21849, and PHY-95-07740, and by research funds provided by Syracuse University.

\section{APPENDIX A: DOUBLE COVER CONICAL SINGULARITY IN THE TRIAD-CUM-CONNECTION FORMULATION OF (2+1)-DIMENSIONAL GRAVITY}

In this appendix we shall consider, in the triad-cum-connection formulation of Einstein gravity (sometimes also called the "first order formalism" or "Cartan formulation"), the $(2+1)$-dimensional spacetime that is obtained as the product of the time axis with the double cover conical singularity of subsection IIC. We first review how this spacetime can be regarded as an exact solution in this formulation. We then demonstrate that if one attempts to approach this spacetime via a specific class of nondegenerate triads, such that the equation of motion relating the connection to the triad is imposed, the connection does not have a limiting value, and its curvature approaches a delta-function rather than zero. Moreover, even neglecting the connection, the sequence of non-degenerate triad fields does not go over as $\gamma \rightarrow 0$ to the triad field of the solution. 
Recall that in the triad-cum-connection formulation of $(2+1)$-dimensional gravity of Refs. 68 71, the fundamental variables are the co-triad $e_{a I}$ and an $\mathrm{SO}(2,1)$ connection $A_{a}^{I}$. We follow the notation of Refs. [69,70] (for these appendices only). Assuming the spacetime to be orientable, the action is

$$
S=\frac{1}{2 \kappa} \int d^{3} x \tilde{\eta}^{a b c} e_{a I} F_{b c}^{I}
$$

where $\tilde{\eta}^{a b c}$ is the Levi-Civita density and $F_{a b}^{I}$ is the curvature of the connection,

$$
F_{a b}^{I}=2 \partial_{[a} A_{b]}^{I}+\epsilon^{I}{ }_{J K} A_{a}^{J} A_{b}^{K}
$$

The field equations are

$$
\begin{aligned}
& F_{a b}^{I}=0, \\
& \mathcal{D}_{[a} e_{b] I}=0,
\end{aligned}
$$

where $\mathcal{D}_{a}$ is the gauge covariant derivative determined by $A_{a}^{I}$,

$$
\mathcal{D}_{a} v_{K}=\partial_{a} v_{K}-\epsilon^{I}{ }_{J K} A_{a}^{J} v_{I}
$$

If the triad is invertible, equations ( $\mathrm{A3b}$ ) can be uniquely solved for the connection. Inserting this solution back into the action (A1) yields the action of the usual metric theory expressed in terms of the triad,

$$
S=\frac{1}{2 \kappa} \int d^{3} x \sqrt{-g} R
$$

We wish to consider the spacetime metric

$$
d s^{2}=-d t^{2}+\left(x^{2}+y^{2}+\gamma\right)\left(d x^{2}+d y^{2}\right)
$$

where $\gamma \geq 0$. For $\gamma=0$ this metric is just the product of the time axis and the double cover conical singularity metric studied in subsection IIC. It is well known that the conical singularity metric can be regarded as a solution to the triad-cum-connection formulation 41]; an explicit connection and triad are given by $A^{I}=0$ and

$$
\begin{aligned}
& e^{0}=d t \\
& e^{1}=x d x-y d y \\
& e^{2}=x d y+y d x
\end{aligned}
$$

Let then $\gamma>0$. We choose a triad compatible with (A6) to be

$$
\begin{aligned}
e^{0} & =d t, \\
e^{1} & =\sqrt{x^{2}+y^{2}+\gamma} d x, \\
e^{2} & =\sqrt{x^{2}+y^{2}+\gamma} d y .
\end{aligned}
$$


Solving (A3b) for the connection yields

$$
A^{0}=\frac{x d y-y d x}{x^{2}+y^{2}+\gamma}
$$

and the curvature form (A2) is then given by

$$
F_{x y}^{0}=\frac{2 \gamma}{\left(x^{2}+y^{2}+\gamma\right)^{2}}
$$

In the limit $\gamma \rightarrow 0, F_{x y}^{0}$ tends to $2 \pi \delta_{2}(x, y)$, as can be verified by a straightforward integration against a test function. Thus, in this limit one does not recover a solution to the connection formulation, but instead one obtains a singular connection with a nonvanishing distributional curvature. Comparing (A1) and (A5) shows that this distributional curvature agrees with the result (2.29) for the Ricci scalar density in the metric formulation.

The failure of the $\gamma \rightarrow 0$ limit to yield a solution to the connection formulation is related to the difference between (A7) and the $\gamma \rightarrow 0$ limit of (A8), which are connected by a singular gauge transformation that changes the "winding number" of the triad around closed loops that encircle the $t$-axis. Indeed this difference of winding number seems to imply that the solution configuration cannot be obtained as the limit of any sequence of non-degenerate configurations. In that sense it would belong to an entirely different component of "history space" than the regular configurations.

\section{APPENDIX B: THE HOURGLASS SPACETIME}

In this appendix we consider the $(2+1)$-dimensional metric

$$
d s^{2}=-d t^{2}+\left(t^{2}+\gamma\right) d \varphi^{2}+d z^{2}
$$

where $t$ and $z$ take all real values, $\varphi$ is periodic with period $\beta>0$, and $\gamma \geq 0$. For $\gamma>0$ the metric is invertible everywhere. For $\gamma=0$, one obtains the hourglass spacetime [42,43]: the regions $t<0$ and $t>0$ of the constant $z$ slices are isometric to past and future causal regions of Misner space [2], but at $t=0$ the metric is degenerate.

For $\gamma>0$, the scalar density $\frac{1}{2} \sqrt{-g} R$ in the chart $(t, \varphi, z)$ is given by

$$
\frac{1}{2} \sqrt{-g} R=\frac{\partial^{2}}{\partial t^{2}}\left(\sqrt{t^{2}+\gamma}\right)
$$

which has at $\gamma \rightarrow 0$ the distributional limit

$$
\frac{1}{2} \sqrt{-g} R=2 \delta(t)
$$

Integrating (B3) over the whole spacetime yields $2 \beta$, which is twice the real part of the corresponding integral for the yarmulke spacetime considered in subsection [IB. From this

point of view, one can envisage the hourglass spacetime as consisting of two copies of the 
yarmulke spacetime, with one flat dimension added: the yarmulkes have been glued together back to back at the singularity, in such a way that the real parts of the actions add and the imaginary parts cancel.

Consider now the triad-cum-connection description. For $\gamma=0$, the metric $(\mathbb{B 1})$ can be regarded as a solution [42,43]: a compatible triad and connection are given by

$$
\begin{aligned}
& e^{0}=d t, \\
& e^{1}=t d \varphi, \\
& e^{2}=d z,
\end{aligned}
$$

and

$$
A^{2}=d \varphi
$$

Suppose then that $\gamma>0$, and consider the triad

$$
\begin{aligned}
& e^{0}=d t, \\
& e^{1}=\sqrt{t^{2}+\gamma} d \varphi, \\
& e^{2}=d z,
\end{aligned}
$$

which clearly gives the metric (B1). When (A3b) holds, the connection compatible with (B5) is given by

$$
A_{\varphi}^{2}=\frac{\partial}{\partial t}\left(\sqrt{t^{2}+\gamma}\right)
$$

and the curvature form by

$$
F_{t \varphi}^{2}=\frac{\partial^{2}}{\partial t^{2}}\left(\sqrt{t^{2}+\gamma}\right)
$$

In the limit $\gamma \rightarrow 0$, we obtain the non-differentiable triad

$$
\begin{aligned}
& e^{0}=d t, \\
& e^{1}=|t| d \varphi, \\
& e^{2}=d z,
\end{aligned}
$$

and the discontinuous connection

$$
A^{2}=\operatorname{sgn}(t) d \varphi
$$

The curvature has the nonvanishing distributional limit

$$
F_{t \varphi}^{2}=2 \delta(t)
$$


Equation (B9) can also be inferred from the non-smooth fields (B8) directly, without going through the regularization [72]. The Thus, although the fields (B8) are compatible with the hourglass spacetime, they cannot be regarded as a solution to the connection formulation. Indeed they seem to belong, as before, to an entirely different component of the space of field configurations. Again, comparing (A1) and (A5) shows that the result (B9) is in agreement with the result (B3) in the metric formulation.

\footnotetext{
${ }^{14}$ We thank Gary Horowitz for this observation, which first brought the hourglass spacetime to our attention.
} 


\section{REFERENCES}

[1] Misner C W, Thorne K S and Wheeler J A 1973 Gravitation (Freeman, San Francisco)

[2] Hawking S W and Ellis G F R 1973 The Large Scale Structure of Space-time (Cambridge University Press, Cambridge)

[3] Wald R M 1984 General Relativity (University of Chicago Press, Chicago)

[4] Geroch R and Traschen J 1987 Phys. Rev. D 361017

[5] Israel W 1966 Nuovo Cimento 44B 1

[6] Hayward G and Louko J 1990 Phys. Rev. D 424032

[7] Sakharov A D 1984 JETP 60214

[8] Dray T, Manogue C A and Tucker R W 1991 Gen. Rel. Grav. 23967

[9] Ellis G, Sumeruk A, Coule D and Hellaby C 1992 Class. Quantum Grav. 91535

[10] Ellis G F R 1992 Gen. Rel. Grav. 241047

[11] Hayward S A 1992 Class. Quantum Grav. 9 1851; erratum 2453

[12] Kossowski M and Kriele M 1993 Class. Quantum Grav. 102363

[13] Dray T, Manogue C A and Tucker R W 1993 Phys. Rev. D 482587 (gr-qc/9303002)

[14] Hellaby C and Dray T 1994 Phys. Rev. D 495096 (gr-qc/9404001)

[15] Embacher F 1995 Phys. Rev. D 516764 (gr-qc/9501004)

[16] Sorkin R D 1990 in Proceedings of the Third Canadian Conference on General Relativity and Relativistic Astrophysics, edited by A Coley, F Cooperstock, and B Tupper (World Scientific, Singapore)

[17] Borde A and Sorkin R D "Causal Cobordism: Topology Change Without Causal Anomalies" (in preparation); Isham C J (unpublished)

[18] Sorkin R D 1986 Phys. Rev. D 33978

[19] Sorkin R D 1986 Int. J. Theor. Phys. 25877

[20] Sorkin R D 1974 Phys. Rev. D 12 385; 1981 Phys. Rev. D 23565 and references therein

[21] Linet B 1985 Gen. Rel. Grav. 171109

[22] Vickers J A G 1987 Class. Quantum Grav. 41

[23] Vickers J A G 1992 Rend. Sem. Mat. Univ. Pol. Torino 501

[24] Tod K P 1994 Class. Quantum Grav. 111331

[25] Fursaev D V and Solodukhin S N 1995 Phys. Rev. D 522133 (hep-th/9501127)

[26] Clarke C J S, Vickers J A and Wilson J P 1996 "Generalised Functions and Distributional Curvature of Cosmic Strings" e-print gr-qc/9605060

[27] Vilenkin A 1981 Phys. Rev. D 23852

[28] Gott R 1985 Astrophys. J. 2824221

[29] Hiscock W 1985 Phys. Rev. D 313288

[30] Deser S, Jackiw R and 't Hooft G 1984 Ann. Phys. (N.Y.) 152220

[31] Deser S and Jackiw R 1984 Ann. Phys. (N.Y.) 153405

[32] Louko J and Whiting B F 1992 Class. Quantum Grav. 9457

[33] Carlip S and Teitelboim C 1995 Class. Quantum Grav. 121699 (gr-qc/9312002)

[34] Carlip S and Teitelboim C 1995 Phys. Rev. D 51622 (gr-qc/9405070) 
[35] Teitelboim C 1995 Phys. Rev. D 514315 (hep-th/9410103)

[36] Futamase T and Garfinkle D 1988 Phys. Rev. D 372086

[37] Anderson A and DeWitt B 1986 Found. Phys. 1691

[38] Harris S G and Dray T 1990 Class. Quantum Grav. 7149

[39] Manogue C A, Copeland E and Dray T 1988 Pramana 30279

[40] Daughton A, Gu Zh-Ch, O'Connor D J, and Sorkin R D "Topology change and field fluctuations in two dimensions" (in preparation)

[41] Horowitz G T 1991a Class. Quantum Grav. 8587

[42] Horowitz G T 1991b Phys. Lett. 258B 91

[43] Horowitz G T 1992 in Proceedings of the sixth Marcel Grossmann meeting on general relativity, edited by H Sato and T Takamura (World Scientific, Singapore)

[44] Gibbons G W and Hawking S W 1992 Commun. Math. Phys. 148345

[45] Bott R 1982 Bull. Am. Math. Soc. 7331

[46] Bombelli L (unpublished)

[47] Reed M and Simon B 1972 Methods of modern mathematical physics (Academic, New York)

[48] Schwartz L 1957 Théorie des Distributions, vol. I (Hermann, Paris)

[49] Spivak M 1975 A comprehensive introduction to differential geometry, Vol. III (Publish or Perish, Boston)

[50] Allendoerfer C A and Weil A 1943 Trans. Am. Math. Soc. 53101

[51] Reinhart B L 1963 Topology 2173

[52] Jee D J 1984 Geometriae Dedicata 15215

[53] Birman G and Nomizu K 1984 Mich. Math. J. 3177

[54] Law P 1992 Rocky Mountain J. Math. 221365

[55] Chern S S 1945 Ann. Math. 46674

[56] Halliwell J J and Hartle J B 1990 Phys. Rev. D 411815

[57] Balasin H and Nachbagauer H 1993 Class. Quantum Grav. 102271

[58] Mazur P O and Mottola E 1990 Nucl. Phys. B341 187

[59] Ashtekar A and Varadarajan M 1994 Phys. Rev. D 504944

[60] Louko J and Whiting B F 1995 Phys. Rev. D 515583 (gr-qc/9411017)

[61] Hawking S W 1982 in Astrophysical Cosmology: Proceedings of the Study Week on Cosmology and Fundamental Physics, edited by H A Brück, G V Coyne and M S Longair (Pontificiae Academiae Scientiarum Scripta Varia, Vatican City)

[62] Hartle J B and Hawking S W 1983 Phys. Rev. D 282960

[63] Halliwell J J and Louko J 1989 Phys. Rev. D 392206

[64] Salgado R, Sorkin R D, and Surya S, in preparation

[65] Bombelli L and Sorkin R D "When are Two Lorentzian Metrics close?" (in preparation)

[66] Lovelock D 1971 J. Math. Phys. 12498

[67] Lovelock D 1972 J. Math. Phys. 13874

[68] Witten E 1988 Nucl. Phys. B311 46 
[69] Ashtekar A 1991 Lectures on Non-Perturbative Canonical Gravity (World Scientific, Singapore) Chapter 17

[70] Romano J D 1993 Gen. Rel. Grav. 25759

[71] Amano K and Higuchi S 1992 Nucl. Phys. B377 218

[72] Horowitz G T 1993 Private communication 